\title{
Improvement in asthma control and inflammation in children undergoing adenotonsillectomy
}

\author{
Jonathan C. Levin', Lisa Gagnon², Xiaoxuan He', Eric D. Baum², David E. Karas² and Geoffrey L. Chupp'
}

BACKGROUND: Observational studies suggest that asthma control improves after adenotonsillectomy, but longitudinal studies that correlate the effect of the procedure on the levels of biomarkers associated with airway inflammation are limited. METHODS: We conducted a longitudinal, observational study on pediatric patients, both with and without asthma, undergoing adenotonsillectomy. Asthma control test (ACT) scores and chitinase activity in the circulation were measured at time of surgery and at 6-mo follow-up.

RESULTS: Sixty-six children with asthma and 64 control subjects were enrolled. Mean ACT scores improved by three points $(P<0.001)$ after 6 mo. 85\% of children with poorly controlled asthma demonstrated an increase in ACT score of at least three points or a decrease in emergency department/urgent care visits, oral corticosteroid courses, or rescue short acting bronchodilator usage. Chitinase activity decreased significantly in asthmatics who improved $(P<0.01)$. Higher chitinase activity levels at baseline were associated with improved asthma control following surgery $(P<0.01)$.

CONCLUSION: In children with high preoperative circulating chitinase activity levels, asthma control and healthcare utilization were significantly improved after adenotonsillecotmy. Chitinase activity decreased after surgery in children with improved control. This suggests that adenotonsillectomy modulates chitinase activity, affecting airway inflammation and improving airway disease.

A sthma is an inflammatory disease of the lower airways that remains a significant healthcare concern for the pediatric population despite public health and pharmacologic advances to control the disease $(1,2)$. Studies have demonstrated a systemic component to asthmatic disease, and that upper airway inflammation may specifically be involved in the pathogenesis of asthma (3-5). Upper airway infections and inflammation are common in the pediatric population; likewise, respiratory infection and allergen exposure are frequently indicated as triggers for asthma exacerbations in this population.

Adenotonsillectomy is one of the most common surgical procedures in children, performed to address upper airway lymphoid hypertrophy, infection, and inflammation (6). Its clinical effect on asthma control has been debated (7). Early observational studies demonstrated that up to $88 \%$ of the patients with asthma experience a significant improvement in symptoms and reduce or eliminate asthma controller or rescue medication usage following adenotonsillectomy (8). A recent retrospective chart review of 93 children with asthma who underwent tonsillectomy for standard indications showed significant improvements in multiple asthma measures including mean hospital visits, systemic steroid administration, asthma medication use, and childhood asthma control test (ACT) scores (9). In addition, a recent cohort study identified children with poorly controlled asthma and referred them for polysomnography; if diagnosed with obstructive sleep apnea, the children were offered adenotonsillectomy. Posttonsillectomy data for 35 children showed a significant improvement from adenotonsillectomy in asthma control, defined by asthma exacerbations, weekly rescue medication usage, asthma symptom score, and forced expiratory volume $\left(\mathrm{FEV}_{1}\right)(10)$. Although there are clear effects of adenotonsillectomy on the upper airway, to our knowledge, the effect of this procedure on markers of airway inflammation has not yet been studied.

Chitinases are a family of hydrolases detectable in the circulation that correlate with inflammation and disease activity in a number of chronic diseases including asthma and adenotonsillar disease (11). These proteins bind to or cleave chitin, the second most abundant polysaccharide in nature and the major structural polymer in cell walls of bacteria and fungi, the shells of crustaceans, and the exoskeletons of arthropods such as cockroaches and dust mites; many of which are common allergic triggers in asthma. Though mammals do not have chitin, they have conserved chitinase proteins to break down chitin from the environment $(11,12)$. There are two major chitinases in humans; chitotriosidase is a true enzyme with the ability to hydrolyze chitin, while YKL-40 is a chitinase-like protein, which has the ability to bind to chitin but not to degrade it (13). The enzymatically active chitinase, chitotriosidase (CHIT1) is overexpressed in adenoid tissue of children undergoing adenotonsillectomy with concurrent chronic rhinosinusitis, otitis media with effusion, and allergic rhinitis compared to subjects without concomitant upper airway disease (14). Additionally, proteins in the chitinase family have been demonstrated as 


\section{Articles $\mid$ Levinet al.}

markers of the systemic component of asthmatic disease. A cross-sectional study of children with allergic and nonallergic asthma showed that chitotriosidase levels were elevated in the serum of individuals with asthma children compared to control subjects (15). YKL-40 has been reported to be elevated in the serum of individuals with asthma and these levels correlate positively with disease severity and airway inflammation (16).

Chitinases may reflect a biological link between upper airway inflammation alleviated by adenotonsillectomy and environmentally triggered lower airway inflammation in the pathogenesis of asthma. This study aimed to enroll large cohorts of pediatric adenotonsillectomy patients with and without asthma, follow asthma control from baseline to postoperative follow-up and record biological activity in the chitinase family that may be associated with disease improvement.

\section{RESULTS}

One hundred and thirty patients undergoing adenotonsillectomy were enrolled, ages $2-18$, over a 13 -mo period. Sixty-six subjects had diagnoses of asthma; 64 subjects did not have a diagnosis of asthma (controls). $75 \%$ of study participants had a primary indication of tonsillar hypertrophy, including symptoms of sleep-disordered breathing, for surgery. Twentythree percentage of participants had a primary indication of recurrent tonsillitis/infection for surgery (Table 1). Compared to controls, children with asthma had higher incidence of expected comorbidities including atopy (history of seasonal allergies and/or eczema) and a history of gastroesophageal reflux disease (GERD) and were more commonly of Hispanic origin (17). Of children $\geq 6$ y of age who completed pulmonary function tests (PFTs), those with asthma had a lower median $\mathrm{FEV}_{1}$, consistent with a physician diagnosis of asthma. Baseline ACT scores ranged from 6 to 27, with a median of 22 (interquartile range: 16-22). Fourty-six children completed the pediatric ACT and another 5 children completed the adult ACT at baseline; 15 children never completed a baseline ACT. Twenty-nine subjects were administered ACT questionnaires following surgery during the follow-up period, rather than prior to surgery. These families completed the ACT prior to follow up by phone or at the follow-up appointment. There was no relation between indication for surgery and baseline ACT score. Thirty-eight out of 66 (58\%) had poorly controlled asthma (ACT $<20, \geq 2$ emergency department (ED)/ urgent care visits in the previous year, $\geq 2$ oral corticosteroid courses in the previous year, or using short acting bronchodilator (SABA) medication more than twice per week in the last month).

Follow-up was achieved in $81 \%$ of the enrolled subjects $(N=105), 58$ subjects with asthma and 47 control subjects. Bloodwork was obtained for 122 participants (94\%) at baseline; 67 parents gave consent (64\% of those achieving followup) to have bloodwork performed postoperatively. Mean time to follow up was 7.0 mo (range: 5-12; SD: 1.5) and was not statistically different between study groups. Follow-up rate was also not statistically different for patient age, gender, race, ethnicity, or indication for tonsillectomy.
Table 1. Baseline demographics, clinical characteristics, and laboratory data of asthma and control cohorts enrolled in the study

\begin{tabular}{|c|c|c|}
\hline Characteristics & Asthmatics & Controls \\
\hline Age, mean (range) & $6.4(2-17)$ & $5.8(2-18)$ \\
\hline Females, $n(\%)$ & $25(38)$ & $33(52)$ \\
\hline Males, $n(\%)$ & $41(62)$ & $31(48)$ \\
\hline \multicolumn{3}{|l|}{ Race, $n(\%)$} \\
\hline White/Caucasian & $54(82)$ & $43(67)$ \\
\hline Black/African American & $11(17)$ & $20(31)$ \\
\hline Other & $1(1)$ & $1(2)$ \\
\hline Hispanic and/or Latino ethnicity, n (\%)* & $32(49)$ & $19(30)$ \\
\hline \multicolumn{3}{|l|}{ Comorbidities } \\
\hline Atopy, $n(\%)^{* *}$ & $49(77)$ & $32(51)$ \\
\hline History of GERD, $n(\%)^{* *}$ & $25(38)$ & $9(14)$ \\
\hline Sinusitis, $n(\%)$ & $22(33)$ & $12(19)$ \\
\hline Second hand smoke exposure, $n(\%)$ & $18(29)$ & $17(27)$ \\
\hline \multicolumn{3}{|l|}{ Weight (described), $n(\%)$} \\
\hline Normal or underweight & $47(71)$ & $51(80)$ \\
\hline Overweight/obese & $19(29)$ & $13(20)$ \\
\hline \multicolumn{3}{|l|}{ Residence } \\
\hline Urban, $n(\%)$ & $24(37)$ & $20(31)$ \\
\hline Suburban, $n(\%)$ & $34(52)$ & $38(60)$ \\
\hline Rural, $n(\%)$ & $7(11)$ & $6(9)$ \\
\hline \multicolumn{3}{|l|}{ Tonsillectomy indication, $n$ (\%) } \\
\hline $\begin{array}{l}\text { Hypertrophy, including obstructive sleep } \\
\text { apnea }\end{array}$ & $46(70)$ & $52(81)$ \\
\hline Chronic/recurrent tonsillitis & $18(27)$ & $12(19)$ \\
\hline FEV1 \% predicted, median* & 93 & $112 \%$ \\
\hline IgE levels (IU/ml), median & 45.7 & 25.4 \\
\hline
\end{tabular}

${ }^{*} P<0.05,{ }^{*} P<0.01$ (Mann-Whitney U-test, Fisher's exact test, or $\chi^{2}$ test). Sixty-six asthmatics, 64 controls. Italic typeface indicates significance $(P<0.05)$. FEV, forced expiratory volume; GERD, gastroesophageal reflux disease; IgE, immunoglobulin E.

Asthma control and asthma-related healthcare utilization were significantly improved after adenotonsillectomy. There was a clinically significant improvement in ACT scores after 6 mo (median increase from 22 to $25, P<0.001$; Figure 1a), and subjects with asthma reported significant decreases in the rate of ED/urgent care visits, oral corticosteroid courses, missed school days due to asthma, and total missed parental work days due to illness (Table 2). In subjects with ACT scores of $<25$ at baseline, the scores increased $\geq 3$ in 24 of 35 children $(69 \%, P<0.001$; Figure 1 b). In addition, 12 of 27 children (44\%) on controller medications for asthma at baseline were no longer on controller medication at follow-up. Two children in the control arm "developed" asthma during the follow-up period. PFT scores did not significantly change for either study group between baseline and follow-up. Subgroup analysis of children with poorly controlled asthma at baseline demonstrated a median increase in ACT score after surgery of 6 $(P=0.02)$. 
a

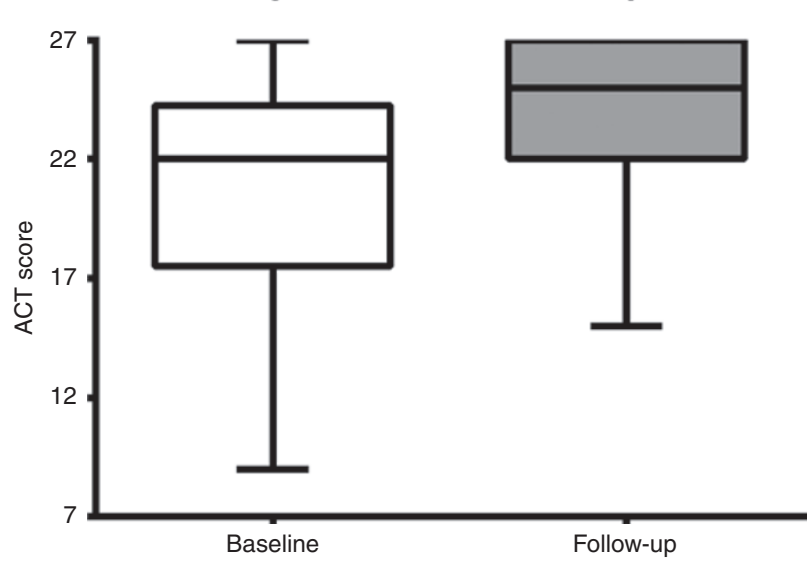

b

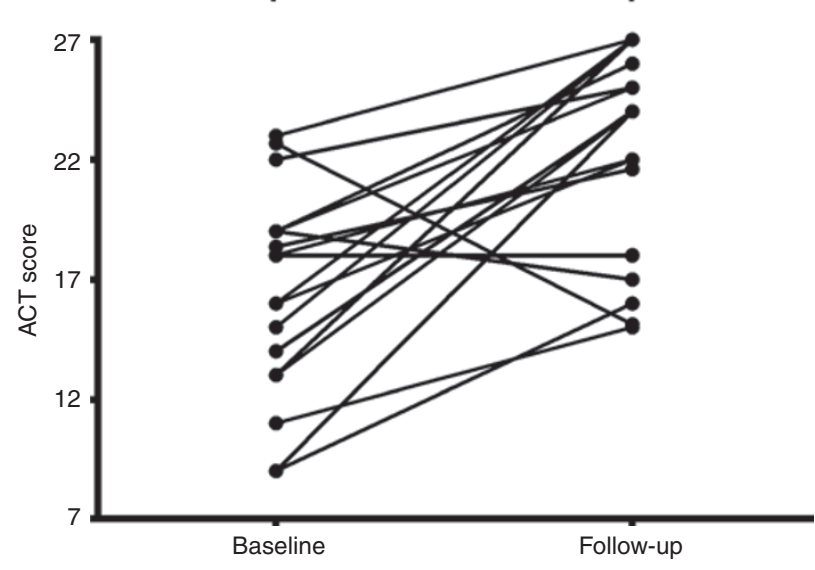

Figure 1. Asthma control test (ACT) scores at baseline and follow-up. (a) ACT scores in all children with asthma. There was an improvement in median scores by 3 postoperatively (Wilcoxon signed rank test), $n=42$. (b) Pairwise changes in ACT score, for baseline scores $<25$. Scores increased $\geq 3$ in 24 of 35 (69\%) children (Wilcoxon signed rank test), $n=18$. Median score displayed with interquartile ranges; error bars represent range of minimum and maximum scores. ${ }^{\dagger} P<0.001$ (Wilcoxon signed rank test).

Among child ACT scores, there were significant pairwise increases in responses to six of the seven questions, including child self-rating of asthma symptoms that day (question 1; $P<0.01$ ), of cough (question 3; $P<0.0001$ ), and of nighttime symptoms (question $4 ; P<0.0001$ ). All three questions regarding parental assessment (questions 5-7; frequency of daytime symptoms, $P=0.0001$; frequency of wheeze, $P<0.01$; and frequency of nighttime symptoms, $P<0.001$ ) also demonstrated significant pairwise increases. Child self-rating of limitations due to asthma did not significantly change in pairwise analysis.

The composite variable of improvement in asthma control was defined by fulfilling one of the following categories: increase in ACT score of 3 or greater, decreased rate of emergency/urgent care visits, a decreased rate of oral corticosteroid courses, or a decrease in rescue SABA usage in the previous month. Of the 48 children with asthma who were not already maximally controlled at baseline (pediatric ACT $<25$, adult ACT $<23$, one or more $\mathrm{ED} /$ urgent care visit in the previous year, one or more oral corticosteroid courses in the last year, or using SABA
Table 2. Change of clinical characteristics from baseline to follow-up in asthma cohort

\begin{tabular}{lcc}
\hline Event frequency per $12 \mathrm{mo}$ & $\begin{array}{c}\text { Baseline } \\
\text { (mean) }\end{array}$ & $\begin{array}{c}\text { Follow-up } \\
\text { (mean) }\end{array}$ \\
\hline Emergency room/urgent care visits for asthma* & 1.88 & 0.40 \\
Oral corticosteroid courses ${ }^{* *}$ & 1.11 & 0.21 \\
Hospitalizations for asthma & 0.09 & 0.00 \\
Missed school days due to asthma* & 3.86 & 2.00 \\
Total missed parental work days* & 2.79 & 1.13 \\
\hline${ }^{*} P<0.05,{ }^{* *} P<0.01$ (Wilcoxon signed rank test). Italic typeface indicates significance \\
$(P<0.05)$.
\end{tabular}

medication in the last month), 36 (75\%) had an improvement in symptoms in at least one category. When limited to the 34 subjects with poorly controlled asthma at baseline, $29(85 \%)$ had an improvement in symptoms in at least one category.

Improvement in the asthma control composite variable did not correlate with baseline patient characteristics including age, years since asthma diagnosis, comorbidities, smoke exposure, indication for adenotonsillectomy, or PFTs. However, analysis of children with poorly controlled asthma at baseline, showed that asthma control improved in all 19 subjects who listed "environment" as a trigger, while control improved in only 10 of $15(67 \%)$ of those who did not list "environment" as a trigger $(P=0.01)$. In addition, improvement in control was significantly associated with a lower baseline ACT score among those with poor control at baseline (a median score of 22 for subjects with improved asthma control vs. a score of 16 for those without improved control, $P<0.05$ ).

Chitinase activity and YKL-40 levels were evaluated at baseline and follow-up. While there was a significant decrease in circulating chitinase activity in children with asthma (median decrease $0.4 \mathrm{nmol} / 1 / \mathrm{ml}{ }^{\star} \mathrm{h}, P<0.01$ ), chitinase activity did not change significantly in control subjects after surgery (median no change, $P=0.83$ ). YKL-40 levels did not change significantly in either study group (Figure $2 \mathrm{~b}$ ).

Among children with asthma, those with improved asthma control demonstrated significantly decreased chitinase activity after adenotonsillectomy $(P=0.001)$, while those without improvement had no significant change in chitinase activity $(P=0.73$; Figure 2a). Baseline chitinase activity was also significantly higher in subjects whose asthma control improved vs. those whose control did not (median 3.5 vs. $2.2 \mathrm{nmol} / \mathrm{l} /$ $\left.\mathrm{ml}{ }^{\star} \mathrm{h}, P<0.01\right)$. These results were also demonstrated in the subgroup of children with poorly controlled asthma at baseline (defined by the composite control variable); chitinase activity significantly decreased when asthma control improved (median decrease $0.9 \mathrm{nmol} / \mathrm{l} / \mathrm{ml}^{\star} \mathrm{h}, P<0.01$ ), but not when control remained unchanged (median no change, $P=1.00$; Figure 2c). Baseline chitinase activity was significantly higher in subjects whose asthma control improved vs. those whose asthma control did not (median 3.4 vs. $1.9 \mathrm{nmol} / \mathrm{l} / \mathrm{ml}{ }^{\star}$ h; $P<0.05$ ). In this subgroup of children with poorly controlled asthma, a baseline chitinase activity $\geq 2.35$ predicted improvement with $82 \%$ sensitivity and $80 \%$ specificity. 


\section{Articles $\mid$ Levin et al.}

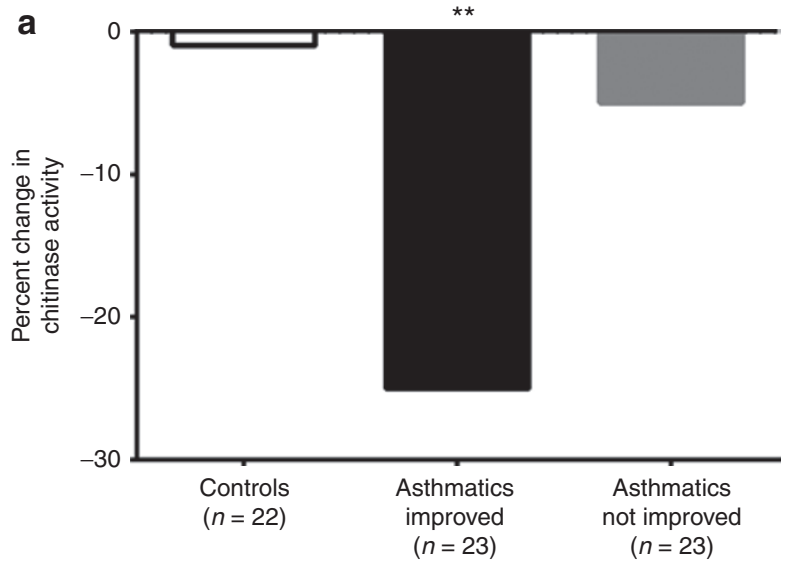

b
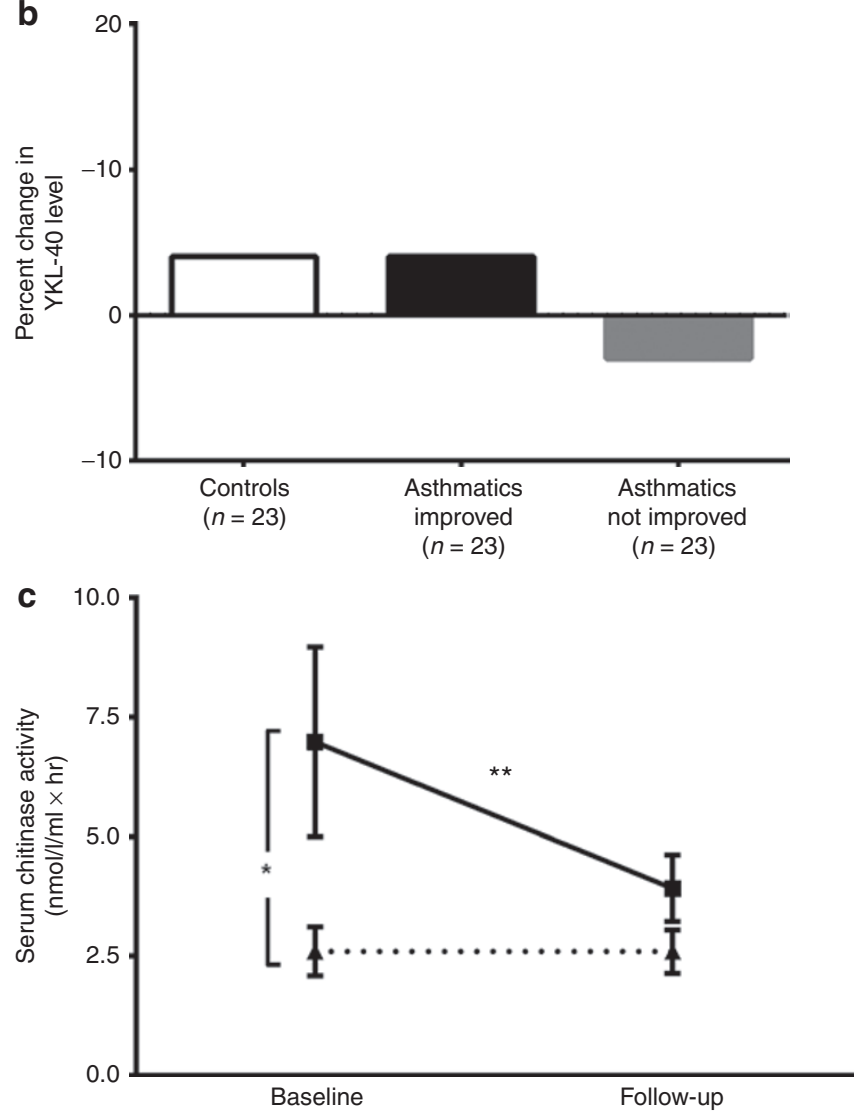

Figure 2. Change in chitinase activity and YKL-40 level over study period. (a) Percent change in chitinase activity. Twenty-two controls, 22 asthmatics with improvement, and 16 asthmatics without improvement. Change in chitinase activity was significant for asthmatics with improvement, but not others (Wilcoxon signed rank test). (b) Percent change in YKL-40 level. Twenty-three controls, 22 asthmatics with improvement, and 18 asthmatics without improvement. Change in chitinase activity was not significant for any group (Wilcoxon signed rank test). (c) Change in chitinase activity in poorly controlled asthmatics. Solid line with squares represents 19 poorly controlled asthmatics who improved, and dotted line with triangles represents three poorly controlled asthmatics who did not improve control. Baseline chitinase activity was significantly higher in children that improved (Mann-Whitney $U$-test), and chitinase activity significantly decreased in children who did not (Wilcoxon signed rank test). Chitinase activity did not significantly change in children who did not improve. ${ }^{*} P<0.05,{ }^{*} P<0.01$. Poorly controlled defined as baseline asthma control test score $<20$, rescue short acting bronchodilator usage $>2$ times/week, emergency room/urgent care visits $\geq 2$ /year, or oral corticosteroid courses $\geq 2$ /year.

\section{DISCUSSION}

Though a number of observational studies have demonstrated a positive clinical effect of adenotonsillectomy on asthma control, these studies have been limited in size and study design (18). To our knowledge, no study has examined the effect of adenostonsillectomy on markers of airway inflammation; we chose to explore chitinases given previous, separate findings linking them to both asthma and adenotonsillectomy $(14,16)$. In this study, we have demonstrated that asthma control and healthcare utilization significantly improve after adenotonsillectomy and that this improvement is associated with a decrease in chitinase activity in the circulation that is not evident in children without asthma. Therefore, while this procedure clearly has effects on upper airway inflammation and mechanics that may contribute to improved asthma control, these results suggest that adenotonsillectomy also modulates airway inflammation in asthma.

For this study, children with asthma undergoing adenotonsillectomy for standard clinical indications were evaluated using both clinical and biological parameters. We used a past physician diagnosis of asthma as our identification criteria, which was supported by the statistically lower $\% \mathrm{FEV}_{1}$ in the asthmatic group. The asthmatic group also had statistically more children of Hispanic or Latino heritage; however, this did not significantly correlate with chitinase activity or YKL-40 levels. A 6-mo follow-up was chosen to minimize natural age-related changes in asthma symptoms, but to provide adequate time for biological changes in airway inflammation and asthma control endpoints to occur. Participants were enrolled year-round to minimize effects of seasonal variability on changes in asthma symptoms.

The study demonstrated that a vast majority of subjects with asthma undergoing adenotonsillectomy reported improvement in symptoms 6 mo after the surgery. $75 \%$ of all children with asthma not already maximally controlled at baseline reported symptom improvement. ACT score, a validated tool to monitor asthma symptoms and severity, increased on average over three points from baseline to postoperative follow-up in all surgical patients with asthma $(19,20)$.

Subgroup analysis of children with poorly controlled asthma at baseline was performed in order to identify a potential benefit of surgical intervention in those with asthma refractory to standard medical therapies. $85 \%$ of children with poorly controlled asthma at baseline reported improved symptom control. ACT score improved in these children by over six points. Response to surgery was also significantly associated with a lower baseline ACT score, suggesting that those with poorly controlled asthma were most likely to see a benefit from the surgery.

One strength of this study is that we have identified a serum biological marker, chitinase activity, that decreases with improvement in asthma after adenotonsillectomy but does not change in subjects without asthma undergoing the procedure. Given the difficulties of performing a randomized controlled trial of surgery for pediatric asthma patients, identifying positive clinical and biological predictors for symptom 
improvement after adenotonsillectomy would help to categorize patients who would most likely benefit from the procedure and further justify a biological effect of the surgery on asthmatic inflammation. We have also shown that higher serum chitinase activity was significantly associated with improvement in disease activity in both the entire cohort of individuals with asthma as well as the subgroup with poorly controlled asthma at baseline. These data suggest that the surgery improves not only clinical asthma symptoms but also has a biological effect on inflammation associated with asthma.

These results suggest that in patients who improve after adenotonsillectomy, tonsillar inflammation and hypertrophy may trigger inflammation in the lower airways; this stress may be represented by report of the environment as a trigger and biologically by elevated serum chitinase activity at baseline. Removing the tonsils may reduce a stressor on the airway, leading to decreased inflammation, lower chitinase activity, and improved asthma control. Chitotriosidase has been shown to have both pro- and anti-inflammatory properties. Whether the enzyme is responding to inflammation in the airways induced by the environment as an attempt at negative feedback or mediating the inflammation occurring in the airways cannot be discerned by this study.

Limitations to the observational study include regression to the mean; however, longitudinal measurement of ACT scores and other measures of asthma control support our findings. Our asthmatic group had a high prevalence of poorly controlled asthma and was thus more severe than population data; however, this may be explained given our starting point of children who were candidates for adenotonsillectomy, and thus, may have been more connected to the medical system or more likely to have concurrent illnesses. Finally, though this study was designed to examine patients undergoing adenotonsillectomy, these results suggest that a prospective trial examining the effect of adenotonsillectomy in patients with poorly controlled asthma with elevated chitinase levels should be considered. Since standardized commercial assays are avaialble to measure chitinase activity and YKL- 40 , there are no limitations to further studies of these biomarkers in tonsillectomy $(15,16)$.

This study demonstrates that a vast majority of children with asthma, and particularly those with poorly controlled asthma, undergoing adenotonsillectomy for standard indications are likely to see an improvement in disease control. Clinical markers associated with response include lower ACT scores at baseline, environmental triggers of asthma, and higher serum chitinase activity at baseline. This information could help identify a subgroup of children with poorly controlled asthma likely to benefit from the surgery, and support the rationale behind adenotonsillectomy as an intervention for asthma.

\section{METHODS}

Pediatric patients ages $2-18$, with and without asthma, who were undergoing adenotonsillectomy for standard indications at Yale New Haven Children's Hospital and North Haven Surgery Center were recruited over a 13-mo period. Informed parental consent and child assent for patients age $\geq 7$ were obtained. All procedures were approved by the Yale University Human Investigation Committee.
On the day of surgery, a study member administered a questionnaire to the subject's parent. Definition of asthma was based on a previous physician diagnosis prior to surgery. The questionnaire consisted of pulmonary history, including history of asthmatic disease; a list of common triggers with examples including infection, allergy, household (dust, smoke, carpet), environment (pollution, cold weather), and exercise; ED or urgent care visits related to asthma; steroid courses; comorbidities including history of sinus disease, gastroesophageal reflux disease, allergic rhinitis, and eczema; medications, including compliance, perception, and frequency of usage in the last $4 \mathrm{wk}$; and days of school missed and parental days of work missed due to the child's asthma and other illnesses. Subjects with asthma also completed an age-appropriate version of the ACT, with the assistance of parents or guardians as needed $(19,20)$. Adult ACT scores were rescaled from maximum 25 to 27 in order to compare to childhood ACT scores, which also have a maximum score of 27. Children were defined as having poorly controlled asthma at baseline by one of the following criteria, based on asthma and ACT guidelines: pediatric or adult $\mathrm{ACT}<20$, two or more $\mathrm{ED} /$ urgent care visits in the previous year, two or more oral corticosteroid courses in the previous year, or using SABA medication more than twice per week in the last month (21). Children age 6 and above completed PFTs if they were able to comply with the instructions given. Intraoperatively, the anesthesiology team obtained venous blood in serum.

Six months following surgery, parents were contacted by telephone or email. Primary study outcome for children with asthma was measured by retesting an ACT score. The follow-up questionnaire was also administered to parents, with rates calculated for variables measuring number of incidences over a given time (emergency/urgent care visits, steroid courses, school and work days missed). Changes were used to calculate a composite variable of improvement in asthma control, with improvement defined as one of the following: improvement in ACT score of 3 or greater (previously shown to be the minimally important difference in score) (22), decreased rate of emergency/urgent care visits, a decreased rate of oral corticosteroid courses, or a decrease in rescue SABA usage in the previous month. PFTs were also repeated for those study subjects with initial testing. Repeat blood samples were drawn at this time as well. Study participants were compensated for completing follow-up.

Serum chitinase activity was determined using a fluorimetric assay as previously described, and reported as $\mathrm{nmol} / \mathrm{ml}^{\star} \mathrm{h}(13,23)$. YKL-40 levels were determined using commercially available enzyme-linked immunosorbent assay (ELISA) kits (MicroVue, Quidel, San Diego, CA), as previously described (16). IgE levels were measured by commercially available services (Healthpoint Diagnostix, Lincolnshire, IL).

Clinical and experimental data were uploaded into the Yale Center for Asthma and Airway Diseases online database. Statistical analyses were performed using SPSS Statistics version 19 software (IBM, Armonk, NY). Nominal data were compared using Fisher's exact test and Pearson's $\chi^{2}$ test; all scaled data were confirmed to be of a nonnormal distribution with the Shapiro-Wilk test for normality; thus, nonparametric methods including Mann-Whitney $U$-test and Wilcoxon signed rank tests were employed. $P$ values $<0.05$ were considered significant.

\section{ACKNOWLEDGMENTS}

We acknowledge Melissa Dziedzic and Wendy Mackey for their help in recruitment of patients for this study. We also acknowledge Maria Koenigs for reviewing an initial draft of the manuscript.

\section{STATEMENT OF FINANCIAL SUPPORT}

This study was supported by R01 HL095390-01, National Heart Lung and Blood Institute, and UL1 RR 024146, National Institutes of Health (Bethesda, MD).

Disclosure: The authors have no financial relationships relevant to this article to disclose. The authors have no conflicts of interest to disclose.

\section{REFERENCES}

1. Akinbami LJ, Moorman JE, Liu X; National Center for Health Statistics (U.S.). Asthma Prevalence, Health Care Use, and Mortality: United States, 2005-2009. Hyattsville, MD: US Department of Health and Human Services, Centers for Disease Control and Prevention, National Center for Health Statistics, 2011. 


\section{Articles | Levin et al.}

2. Akinbami L. The state of childhood asthma, United States, 1980-2005. Adv Data 2006:1-24.

3. Bjermer L. Time for a paradigm shift in asthma treatment: from relieving bronchospasm to controlling systemic inflammation. J Allergy Clin Immunol 2007;120:1269-75.

4. Lemanske RF Jr, Dick EC, Swenson CA, Vrtis RF, Busse WW. Rhinovirus upper respiratory infection increases airway hyperreactivity and late asthmatic reactions. J Clin Invest 1989;83:1-10.

5. Braunstahl GJ. The unified immune system: respiratory tract-nasobronchial interaction mechanisms in allergic airway disease. J Allergy Clin Immunol 2005;115:142-8.

6. Baum ED. Tonsillectomy and adenoidectomy and myringotomy with tube insertion. Pediatr Rev 2010;31:417-25; quiz 426.

7. Garabedian EN, Cotin G, Grimfeld A, Beaufrere P, Maillet J. [Effect of amygdalectomy on asthmatic and allergic children. Study apropos of 55 cases]. Ann Otolaryngol Chir Cervicofac 1986;103:589-95.

8. Saito H, Asakura K, Hata M, Kataura A, Morimoto K. Does adenotonsillectomy affect the course of bronchial asthma and nasal allergy? Acta Otolaryngol Suppl 1996;523:212-5.

9. Busino RS, Quraishi HA, Aguila HA, Montalvo E, Connelly P. The impact of adenotonsillectomy on asthma in children. Laryngoscope 2010;120 Suppl 4:S221.

10. Kheirandish-Gozal L, Dayyat EA, Eid NS, Morton RL, Gozal D. Obstructive sleep apnea in poorly controlled asthmatic children: effect of adenotonsillectomy. Pediatr Pulmonol 2011;46:913-8.

11. Ober C, Chupp GL. The chitinase and chitinase-like proteins: a review of genetic and functional studies in asthma and immune-mediated diseases. Curr Opin Allergy Clin Immunol 2009;9:401-8.

12. Wills-Karp M, Karp CL. Chitin checking-novel insights into asthma. N Engl J Med 2004;351:1455-7.
13. Seibold MA, Donnelly S, Solon M, et al. Chitotriosidase is the primary active chitinase in the human lung and is modulated by genotype and smoking habit. J Allergy Clin Immunol 2008;122:944-50.e3.

14. Heo KW, Hur DY, Park SK, Yang YI, Kwak HH, Kim TY. Expression of chitinases in hypertrophied adenoids of children. Otolaryngol Head Neck Surg 2011;145:660-5.

15. Bargagli E, Olivieri C, Margollicci M, et al. Serum chitotriosidase levels in patients with allergic and non-allergic asthma. Respiration 2010;79:437-8.

16. Chupp GL, Lee CG, Jarjour N, et al. A chitinase-like protein in the lung and circulation of patients with severe asthma. N Engl J Med 2007;357:2016-27.

17. Wood PR, Hill VL. Practical management of asthma. Pediatr Rev 2009;30:375-85; quiz 385.

18. Kaiser P. Obstructive sleep apnea in poorly controlled asthmatic children: effect of adenotonsillectomy. Pediatr Pulmonol 2012;47:311; discussion $312-4$.

19. Nathan RA, Sorkness CA, Kosinski M, et al. Development of the asthma control test: a survey for assessing asthma control. J Allergy Clin Immunol 2004;113:59-65.

20. Liu AH, Zeiger R, Sorkness C, et al. Development and cross-sectional validation of the Childhood Asthma Control Test. J Allergy Clin Immunol 2007;119:817-25.

21. Expert Panel Report 3 (EPR-3): Guidelines for the Diagnosis and Management of Asthma-Summary Report 2007. J Allergy Clin Immunol 2007;120:S94-138.

22. Schatz M, Kosinski M, Yarlas AS, Hanlon J, Watson ME, Jhingran P. The minimally important difference of the Asthma Control Test. J Allergy Clin Immunol 2009;124:719-23.e1.

23. Hollak CE, van Weely S, van Oers MH, Aerts JM. Marked elevation of plasma chitotriosidase activity. A novel hallmark of Gaucher disease. J Clin Invest 1994;93:1288-92. 